\title{
CYSTIC MENINGIOMAS: A RADIOLOGICAL AND HISTOLOGICAL STUDY AND PRE OPERATIVE DIAGNOSTIC ERRORS
}

\author{
Jaideep Chandra, ${ }^{1}$ Raj Kumar $\mathrm{KC}^{1}$
}

\begin{abstract}
INTRODUCTION

Meningiomas are solid neoplasms, cysts are extremely rare, diagnostically elusive, and behave differently. Therefore, a study was initiated comparing our cases with various series. This is the second largest series so far reported. We tried to hypothesize how to eliminate diagnostic errors in this study, while few interesting points were discovered.
\end{abstract}

\section{MATERIAL AND METHODS}

All meningiomas operated in the last seventeen years were reviewed. It was a retrospective study. Diagnostic errors, clinical behaviour and histopathology were reviewed. Planning of surgery and prognostication demands that the diagnosis be established pre-operatively and since these tumours may mimic other brain tumour types on radiology, factors that may lead to better diagnostic accuracy were tried to be established. Categorization of the tumours with different cyst types according to various classifications was done. The follow up period was two months to ten years.

\section{RESULTS}

Out of 530 meningiomas, we found 20 with cystic changes.. There mean age was 47.5 years. The commonest symptoms were raised intra cranial pressure, hemiparesis, memory loss, behavioral changes, vision loss, papilloedem and cranial nerve palsies. The most common histological variety was meningothelial meningioma. The incidence of high grade or malignant tumors was $30 \%$. Based on radiology, the highest diagnostic accuracy was achieved in $62.5 \%$ cases only.

\section{CONCLUSION}

Cystic meningiomas continue to pose diagnostic challenges. Multi-planar contrast magnetic resonance imaging (MRI) will help in establishing accurate diagnosis, it reveals cysts missed on computed tomography (CT) scans. This would lead to accurate prognostication and better surgical planning with the aim of preventing recurrences.

KEYWORDS Cystic meningiomas, diagnostic errors, histology, radiology

1. Department of Surgery (Neurosurgery), Universal College of Medical Sciences, Bhairahawa, Nepal

DOI: https://doi.org/10.3126/jucms.v7i1.24681

\author{
For Correspondence \\ Dr. Jaideep Chandra \\ Department of Surgery (Neurosurgery) \\ Universal College of Medical Sciences \\ Bhairahawa, Nepal \\ Email:jaideep999@gmail.com
}




\section{INTRODUCTION}

Meningiomas are usually benign tumours accounting for 13 $18 \%$ of all intracranial neoplasms. ${ }^{1}$ Cystic changes associated with these are rare and range from $1.6-7 \%$ in various surgical series. ${ }^{2}$ Worthy et $\mathrm{al}^{3}$ reported atypical radiological findings in as many as $15 \%$ including cystic changes; these cystic changes have been classified by Rengachary ${ }^{4}$ in two, Ramos ${ }^{5}$ and Inoue et $\mathrm{al}^{6}$ in three, Nauta et $\mathrm{al}^{7}$ in four and Worthington et $\mathrm{al}^{8}$ in five types. Even with CT and MRI scans there may be diagnostic confusion and they may be mistaken for malignant gliomas, metastasis, haemangioblastomas, intracranial abscess and lymphomas. ${ }^{2}$ More than 240 cases now appear in literature, the largest series reported by Fortuna et $\mathrm{al}^{9}$ of 22 cases, followed by Pinna et $\mathrm{al}^{10}$ of 18 cases, Sridhar et $\mathrm{al}^{11}$ of 17 and Ramos et $\mathrm{al}^{5}$ of 15 cases.

\section{MATERIAL AND METHODS}

The data from patients of all meningiomas, operated since June, 1998 upto June, 2015 in Sanjay Gandhi Post Graduate Institute of Medical Sciences and Research, Lucknow U.P. India was collected and reviewed. It was a retrospective collection of data from the patients records and they were compiled and analysed. The follow up period was two months to ten years. In this study only meningiomas with cysts were included. It was a cross sectional retrospective data analysis, solid tumours were excluded from the study. The subset of meningiomas with associated cysts were narrowed down for their unique characteristics which are hereby discussed.

\section{RESULTS}

Out of 530 patients of meningiomas, we found 20 patients with cystic changes. There mean age was 47.5 years, and range from seven months to Eighty two years. Mean duration of symptoms was from nine months to four years.

The commonest symptoms were those of raised intra cranial pressure (ICP) Fifteen (75\%), hemiparesis was seen in Eight $(40 \%)$; recent memory loss in Four $(20 \%)$, behavioral changes in Four $(20 \%)$, and vision defects in Four $(20 \%)$. Total vision loss also in Four (20\%), and papilloedema in Eight (40\%). Cranial nerve palsies seen were upper motor neuron type facial paresis in Six (30\%), and VI nerve involvement in Two $(10 \%)$ and lower cranial nerve involvement in One $(5 \%)$. The presentation according to symptoms is shown in table 1 .

Table 1. Symptoms

\begin{tabular}{lc}
\hline Symptoms & $\begin{array}{c}\text { Patients with } \\
\text { meningioma }\end{array}$ \\
\hline Raised ICP & 15 \\
Hemiparesis & 8 \\
Memory Loss & 4 \\
Behavioral Changes & 4 \\
Vision Defects & 4 \\
Cranial Nerve Palsies & 9 \\
\hline
\end{tabular}

Sixteen patients had CT scans in eight of these MRI was also available. Four patients had only MRI scans.

\section{Radiology, histology and surgical findings}

Hypo-dense tumours with peripheral rim enhancement was seen in four cases and were tumour locations were: one middle cranial fossa base; two parafalcine, and one falco-tentorial. peritumoural edema was extensive in all. Pre-op diagnosis was of a glioma or abscess. Surgical findings, however, due to good cleavage plane, and owing to dural attachments shifted the diagnosis in favour of meningiomas. Two of these were malignant on histopatholgy. Four cases had large hypo-dense tumours with mural enhancing nodule, all located in the temporal lobe. All were diagnosed as gliomas but again due to good cleavage from the surrounding brain, meningiomas were suspected during surgery. The histology was meningothelial in two whereas fibrous and atypical in one each.

Four cases had iso-dense tumours with peripheral cysts and all were correctly diagnosed as meningiomas. In all but one the cyst was peripheral but within the tumour capsule, in one it was extra-tumoural. The histology was meningothelial in three and with microcystic changes in one. Two patients had hypo-dense tumours with irregular contrast enhancement. During surgery the tumours were found to be filled with cysts and histology revealed a microcystic changes in one and a meningothelial meningioma in the other.

One patient had a hypo-dense tumour with peripheral calcification in the left CP angle. Since it was extra axial (Figure 1) a meningioma was suspected and removed. Cysts were filled with xantho-chromic fluid and histology revealed a fibrous meningioma. Two patients had hypo-intense tumours with massive peri-lesional edema and irregular contrast uptake; both suspected of being gliomas both before and during surgery and turned out to be "Collision tumours" with gliomatous and mengiomatous components.

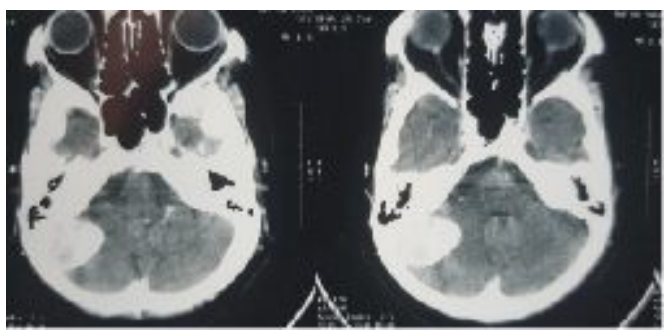

Figure 1. Hypo-dense tumor with calcifiaction

Two patients had large cysts, minimal edema and a solid nodule towards the convexity, suspected as gliomas both pre and peri operatively as xantho-chromic fluid was seen within the tumour during surgery. Both turned out to be meningothelial meningiomas. Two patients had iso-dense tumours with dural attachments seen, peripheral intra tumoural cysts were present in both. One was associated with 
massive edema. Both correctly diagnosed as meningiomas. (Figure 2).

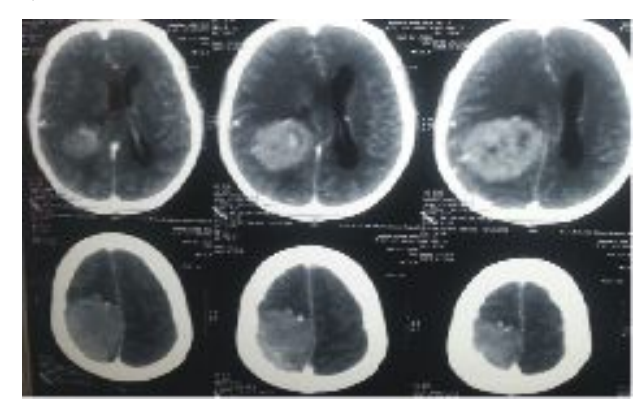

Figure 2. Massive oedema

Overall, peripheral enhancement was seen in four cases Nauta's type I. Two were malignant. Associated intraparenchymal cysts were seen in four cases- Nauta's type III. Four cases were with large cystic appearance and a mural nodule Nauta's type II. Three cases were with multiple cysts both peripheral and central and three cases with peripheral cysts Nauta's type II. Four cases were with both intra and extra tumoural cysts.

Table 2. Distribution of cases according to Nauta's classification

\begin{tabular}{lc}
\hline Classification & Number \\
\hline Nautas Type 1 & 6 \\
Nautas Type 2 & 5 \\
Nautas Type 3 & 2 \\
Combination of Nautas Type 2 and 3 & 2 \\
Combination of Nautas Type 2 and 4 & 2 \\
Multicystictumours not seen in Nauta's & 3 \\
\hline
\end{tabular}

A correct pre operative diagnosis was made in only four cases with CT scans and in one another it was considered in the differential diagnosis thus in five of eight $(62.5 \%)$ patients with only CT scans were correctly diagnosed preoperatively. In patients with only MRI scans, a correct diagnosis was made in two of four cases $(50 \%)$. Further in those with both, a correct diagnosis was made in four of eight cases $(50 \%)$ as shown in table 2. Based on surgical findings, a correct diagnosis was made in 14 patients. In the remaining six, even, the surgical findings did not prompt a differential diagnosis of a meningioma.

Table 3. Diagnostic accuracy with preoperative imaging with CT scan/MRI scan/Both CT and MRI scan

\begin{tabular}{lc}
\hline Pre-operative Imaging & Percentage \\
\hline CT scan only & $62.5 \%$ \\
MRI scan only & $50 \%$ \\
Both CT and MRI scan & $50 \%$ \\
\hline
\end{tabular}

\section{HISTOLOGY}

Based on the histology the distribution of the histologic varieties is shown in table 3 and based on the location the distribution of the cases is shown in table 5 .

\section{Table 4. Histological distribution of cases}

\begin{tabular}{lc}
\hline Histological distribution & Number \\
\hline Meningiothelial Meningioma & 9 \\
Fibrous Meningioma & 2 \\
Malignant Meningioma & 3 \\
Atypical Meningioma & 2 \\
Transitional Meningioma & 1 \\
Myxomatous Meningioma & 1 \\
Collision Tumours & 2 \\
\hline
\end{tabular}

Table 5. According to location

\begin{tabular}{lc}
\hline Location & Number \\
\hline Convexity & 8 \\
Falco Tentorial & 2 \\
Para Falcine & 3 \\
Petrous & 2 \\
Pterional & 3 \\
Middle Cranial Fossa Base & 2 \\
\hline
\end{tabular}

\section{Follow up details}

Follow up range was two months to ten years with a mean of twelve months. Two patients were lost to follow up. One infant was seen eight years after surgery and was found to be tumour free but had a grade IV hemiparesis. Of the two patients with collision tumours, one expired a few months after discharge; the other completed his radio therapy and was tumour free at one year of follow up. Another patient with malignant histology completed his radiotherapy and was tumour free at six months of follow up. One patient with atypical histology died a year later due to unrelated illness. One with malignant histology had a stormy post operative phase and was lost to follow up. One patient with atypical histology was operated for two recurrences, she was earliest operated in 1984 and the details of her first surgery were not available. She was operated upon twice for recurrences in a span of seventeen months.

Other patients were symptom free except for unresolved visual field defects in two patients and VII ${ }^{\text {th }}$ and VIII ${ }^{\text {th }}$ nerve involvement in one (petrous location). For the purpose of study, all patients were contacted and turned up for follow up except the one who was lost to follow up and the two who died.

It was seen that out of all other cases of meningiomas those associated with cysts on radiology were incorrectly diagnosed before or even during surgery. Only histology proved the real nature of the tumour. The use of MRI improves the chances of discovering cysts missed by CT scans, especially, if multi- 
planar imaging is used. It was also seen that the incidence of atypical and malignant tumour grades were seen in a high percentage of cases in meningiomas having cystic changes. After the review of all classifications, it was also seen that most of the classifications were made with CT scans as the diagnostic tool, but with MRI scans we found cyst configurations that were not previously classified by any author thus far.

\section{DISCUSSION}

Comparison of our own cases was done with literature review of the histological types and clinical behavior. The confounding variable here may be the location of the tumour and operating surgeons' skill when tumours are removed with relevance to post-operative results but the study does not compare the outcome, thus eliminating these variables and biases. This study compares the regrowth of histologically malignant variety and the frequency with which they are found in this cystic meningioma subset.

Bouchut first described cysts associated with meninngiomas in 1928. Cushing and Eisenhardt described thirteen cases of cysts associated with meningiomas. Various classifications were published: Rengachary et $\mathrm{al}^{4}$ described two types I) intraand ii) extra tumoural. Ramos et al $^{5}$ classified them into three types based on CT scan findings of cyst locations: Intra tumoural-I, peritumoural-P, and associated type A. I was subdivided into three types : i) small cyst in the centre of the tumour. ii) multiple central cysts; iii) large cyst surrounded by a rim of tumour. Inoue et $\mathrm{al}^{6}$ described three types; i) peripheral cysts with in tumour;ii) a peripheral cyst with enhancing walls; iii) a peripheral cyst with non enhancing walls. Nauta et $\mathrm{al}^{7}$ described four types;i) central cyst with tumour all around. ii) peripheral cyst with thin tumour walls. iii) peritumoural cysts with gliotic walls;iv)cyst bordered by tumour and arachnoid-at the tumour-brain interface. Worthinton et $\mathrm{al}^{8}$ described a fifth type in which the cyst wall was composed of fibrous tissue and tumour cells but they did not stipulate the relation of the cyst with the tumour.

Mechanisms of formation of cyst are hypothesized to arise from; central ischaemic necrosis. ${ }^{2}$ Secretory cells are recognized in some tumours to be responsible for cyst formation. ${ }^{6}$ Microcystic degeneration is responsible for the formation of cysts in a few tumours. ${ }^{11}$ Intratumoural haemorrhage may account for cyst formation in a some tumours. ${ }^{12}$ Myxomatous change was found in one of our cases and intratumoural haemorrhage was seen in three. Confluence of microcysts may lead to formation of larger cysts. ${ }^{11-12}$ Two cases of micro-cystic changes were seen in our series. Extratumoural cysts may result from glial secretion or proliferation, this is known to occur in adjacent brain in response to the presence of a tumour. ${ }^{2}$ Loculation of CSF may result in the formation of Nautas type IV cyst, found in three of our cases. Demyelination of adjacent brain leads to the formation of Nautas type III cyst. ${ }^{11}$ Association with gliomas may be responsible for the formation of such cysts. ${ }^{13}$ We had two such cases.

The most frequent histological variant is meningothelial, ${ }^{9}$ seen in nine of our cases. Meningiomas are rare in infancyaccounting for less than $1-2 \%$ of all intracranial neoplasms, ${ }^{12}$ but the incidence of cystic meningiomas is higher in children$12.5 \%$; with the incidence of cyst formation as high as 55$60 \%$ in those under one year of age, with fibrous histology being the commonest. ${ }^{14}$ We had an unusually high incidence of malignant or high grade tumours. Fortuna et $\mathrm{al}^{9}$ had a $2 \%$ malignancy rate, Sridhar et $\mathrm{al}^{11}$ had $5.9 \%$ and Ramos et $\mathrm{al}^{5}$ had $6.7 \%$ malignancy rates. Worthignton ${ }^{8}$, Dell ${ }^{15}$, Parisi ${ }^{16}$, and Pinna $^{10}$, did not report a single malignant variant in their respective series. In our series, we had two malignant, two collision tumours and two cases of atypical meningiomas accounting for $30 \%$; thus the highest rate of malignant tumours associated with cystic changes reported so far.

Intra tumoural haemorrhage in meningiomas is exceedingly rare. $^{17}$ This was seen in three of our cases; it helps in corroborating the theories of cyst formation and also points to the frequency of malignant change responsible for formation of cysts in these tumours. Various authors have quoted a diagnostic accuracy of $30-80 \%$ based on CT/MRI/ Angiography. ${ }^{6,10}$ Only Wasenko et $\mathrm{al}^{18}$ based on MRI had a $100 \%$ accuracy in diagnosing these tumours pre-operatively. The important differentials are pleomorphic xanthoastrocytomas, malignant gliomas, glio-sarcomas, metastasis and lymphomas. We agree with Ferrante et $\mathrm{al}^{19}$ and Pinna et $\mathrm{al}^{10}$ in that a $100 \%$ pre-operative diagnostic accuracy may not be possible.

\section{CONCLUSION}

Cystic meningiomas are intriguing varieties of a common intra cranial neoplasms. Usually, when we see a tumour with cystic changes, it is considered to be a high grade and malignant tumour with a totally different approach being taken for surgery, post operative treatment, planning and prognostication. However, meningiomas are benign and even with malignant histology they are curable with total excision and radiotherapy as compared to glioblastomas which do not give a lifespan of greater than two years - despite all modalities of treatment- from the time of their diagnosis. Multi-planar gadolinium enhanced imaging may help to increase the index of suspicion in these elusive tumour variants. It is important to recognize them in order to prevent recurrence of these benign tumours, as all of the cyst wall needs to be excised especially in Nautas types I and II. In types III and IV the cyst wall towards the brain may be left behind preventing unnecessary neurologic deficits.. Therefore, it is important that these be kept in mind in making differential diagnosis when we see tumours attatched to dural bases. Use of multi planar gadolinium enhanced radiology as a preoperative diagnostic tool will help increase diagnostic accuracy. 


\section{ACKNOWLEDGEMENTS}

I thank Professor Deepu Banerji and Professor Sanjay Behari. for helping me and guiding me throughout this study.

\section{CONFLICT OF INTERESTS}

None.

\section{REFERENCES}

1. Al Mefty, Ossama: Meningiomas. 1991 Raven Press.

2. CarvalhoGustavao A., Peter Vorkapic, Gabriela Biewener, Majid Samii: Cystic Meningiomas resembling glial tumours. Surgical Neurology 1997; 47:284-290.

3. Worthy S.A., A. Gholkar, P.G.Ince: Unusual C.T. appearances with supra-tentorialmeningiomas. British Journal Of Neurosurgery $1995 ; 9 ; 815-817$.

4. Rengachary S., Batnizki S., Kepes JJ., Morantz RA., O' Boynick P., Watanabe I.: Neurosurgery 4:107-114.

5. Ramos Jr F., Ba Zeze V., Velut S., Jan M.: Cystic Meningiomas. J. Neuroradiology 14:271-286.

6. Inoue T., Kuramatsu C., Sawada K., Matsushima T.: Recurrent Cystic Meningioma. Surgical Neurology 1986, 26:399-404.

7. Nauta H.J.W., W.S. Tucker, W.J.Horseley, J.M.Bilbao, C. Gonsalves: Xanthochromic cysts associated with meningiomas: Journal of Neurology, Neurosurgery, Psychiatry, 1979. 42:529-535.

8. Worthington Curtis, Jean-Louis Caron, Dennis Melanson, Richard Leblanc: Meningioma Cysts. Neurology, 1985, 35.12.1720-1724.

9. Fortuna A., L. Ferrante, M. Acqui, G. Guglielmi, L.Nastronardi: Cystic Meningiomas. ActaNeurochirurgica (1988) 90 ;23-30.

10. Pinna Giampietro, Alberto Beltramello, Paolo Buffatti, BiancarnoSignorini, Romano Colombari, Albino Bricolo, Giuseppe Dalle Ore: Cystic Meningiomas An update. Surgical Neurology 1986;26: 441-452.

11. Sridhar K., R. Ravi, B. Ramamurthy, M.C. Vasudevan: Cystic Meningiomas. Surgical Neurology 1995; 43:235-239.

12. Amano Keiichi, Naohisa Miura, Yasuhiko Tajika, Kuniaiki Matsumori, Osami Kubo, Naotoshi Kobayashi, Koiti Kitamura: Cytic Meningiomas in a 10 month old infant. J neurosurgery 1980; 52:829-833.

13. Maiuri F., DenoBenvenuti, Maria R. De Simone, SossioCirillo, Giuseppe Corriero, Arcangelo Giamundo: Cystic lesions associated with meningiomas. Surgical Neurology 1986; 26: 591-597.

14. GenyaOdake: Cystic Meningiomas: A Report of three patients. Neurosurgery 1992;30: 935-940.
15. Dell Stephen, RamaiahGanti, Abe Steinberger, James Mc Murty: Cystic Meningiomas ; A clinico-radiological study. J. Neurosurgery $1982 ; 57 ; 8-13$.

16. Parisi Giuseppe, Rosaria Tropea, Salvatore Giuffrida, Maria Lombardo, Francisco Giuffre:CysticMeningiomas. J. Neurosurgery 1986; 64:35-38.

17. Modesti M. Luciano, Eugene F. Binet, George H Collins: Meningiomas causing spontaneous intracranial haematomas. J. Neurosurgery 1976;45: 437-441.

18. Wasenko John J., Hochhauser, Edward G. Stopa, Jeffrey A. Winfield: Cystic Meningiomas MR charecteristics and surgical correlations. AJNR Nov 1994; 15:1959-1965.

19. Ferrante L., M. Acqui, P. Lunardi, R. Qasho and A. Fortuna: MRI in the diagnosis of Cystic Meningiomas: Surgical Implications ActaNeurochirurgica 1997;139:8-11. 